\title{
Free dihedral actions on abelian varieties
}

\author{
B. Aguiló VIDAL ${ }^{1}$ (iD) \\ 1 Departamento de Matemáticas, \\ Facultad de Ciencias, Universidad de \\ Chile, Las Palmeras 3425, Ñuñoa, \\ Santiago, Chile. \\ bruno.aguilo@ug.uchile.cl
}

\begin{abstract}
We give a simple construction for hyperelliptic varieties, defined as the quotient of a complex torus by the action of a finite group $G$ that contains no translations and acts freely, with $G$ any dihedral group. This generalizes a construction given by Catanese and Demleitner for $D_{4}$ in dimension three.

\section{RESUMEN}

Damos una construcción simple de variedades hiperelípticas, definidas como el cociente de un toro complejo por la acción de un grupo finito $G$ que no contiene traslaciones y actúa libremente, con $G$ cualquier grupo diedral. Esto generaliza la construcción de Catanese y Demleitner para $D_{4}$ en dimensión tres.
\end{abstract}

Keywords and Phrases: Abelian varieties, dihedral group, free action.

2020 AMS Mathematics Subject Classification: 14K99, 14L30. 


\section{Introduction}

A Generalized Hyperelliptic Manifold $X$ is defined as a quotient $X=T / G$ of a complex torus $T$ by the free action of a finite group $G$ which contains no translations. The manifold $X$ is called a Generalized Hyperelliptic Variety if the torus $\mathrm{T}$ is also projective, i.e., it is an Abelian variety. These have Kodaira dimension zero, as Mistretta showed it to be the case for any étale finite quotient of a torus [5]. Furthermore, these type of manifolds are Kähler and their fundamental group is given by the group of complex affine transformations of $T$ which are lifts of transformations of the group $G$, as stated by Catanese and Corvaja in [2].

Uchida and Yoshihara showed that the only non Abelian group that gives such an action in dimension three is the dihedral group $D_{4}$ of order 8 [6]. Later, Catanese and Demleitner gave a simple and explicit construction for that action [4] and completed the characterization of three-dimensional hyperelliptic manifolds [3]. Some authors have worked with these objects in higher dimension as well. For example, Auffarth and Lucchini Arteche showed that they can be constructed using any finite Abelian group, and that there are simple ways to construct some varieties using non Abelian groups [1], all of these formed as products of manifolds of lower dimension. However, not much is known about hyperelliptic manifolds of dimension greater than 3, and a characterization of these manifolds is far from completed.

The purpose of this note is to help with the understanding of Generalized Hyperelliptic Varieties in higher dimension, showing that Catanese and Demleitner's construction is actually generalizable (in quite a natural way) to every odd dimension. Specifically, for every $n \in \mathbb{N}$ we give a Generalized Hyperelliptic Variety of dimension $2 n+1$ defined by the action of the dihedral group $D_{4 n}$ of order $8 n$ acting on a family of Abelian varieties, from which the construction by Catanese and Demleitner remains as the particular case for $n=1$, and we end with a simple corollary that explains how this allows us to create this type of varieties using any dihedral group.

\section{The construction}

Let $E, E^{\prime}$ be any two elliptic curves,

$$
E=\mathbb{C} /(\mathbb{Z}+\mathbb{Z} \tau), E^{\prime}=\mathbb{C} /\left(\mathbb{Z}+\mathbb{Z} \tau^{\prime}\right)
$$

Now, for $n \in \mathbb{N}$ set $A^{\prime}:=E^{2 n} \times E^{\prime}$ and $A:=A^{\prime} /\langle w\rangle$, where $w:=(1 / 2,1 / 2, \ldots, 1 / 2,0)$.

Theorem 2.1. The Abelian Variety A admits a free action with no translations of the dihedral group $D_{4 n}$ of order $8 n$. 
Proof. First, let us recall that for $k \in \mathbb{N}$, the dihedral group of order $2 k$ is defined as

$$
D_{k}:=\left\langle r, s \mid r^{k}=1, s^{2}=1,(r s)^{2}=1\right\rangle
$$

So, in order to prove the result, we need to find automorphisms of $A$ with the required characteristics, that satisfy the relations described above. And for that, we will use automorphisms of $A^{\prime}$ that descends to those of $A$ in a useful way. Now, set, for $z:=\left(z_{1}, z_{2}, \ldots, z_{2 n}, z_{2 n+1}\right) \in A^{\prime}$, the following linear automorphisms:

$$
\begin{aligned}
R(z) & :=\left(-z_{2 n}, z_{1}, \ldots, z_{2 n-1}, z_{2 n+1}\right) \\
S(z) & :=\left(-z_{2 n},-z_{2 n-1}, \ldots,-z_{2},-z_{1},-z_{2 n+1}\right)
\end{aligned}
$$

and with these, we define the following automorphisms of $A^{\prime}$ :

$$
\begin{aligned}
& r(z):=R(z)+\left(0, \ldots, 0, \frac{1}{4 n}\right), \\
& s(z):=S(z)+\left(b_{1}, b_{2}, \ldots, b_{2 n}, 0\right),
\end{aligned}
$$

where, for $i=1, \ldots, n, b_{2 i-1}:=1 / 2+\tau / 2$ and $b_{2 i}:=\tau / 2$.

Step 1. It is easy to verify that $r$ and $R$ have order exactly $4 n$ on $A^{\prime}$. Also, since $R$ is linear and $R(w)=w$, then $r(z+w)=r(z)+w$, and so $r$ descends to an automorphism of $A$ of order exactly $4 n$. Moreover, any power $r^{j}$, for $0<j<4 n$, acts freely on $A$ since the $(2 n+1)$-th coordinate of $r^{j}(z)$ equals $z_{2 n+1}+\frac{j}{4 n}$ (and so is distinct from $z_{2 n+1}$ ). Also, clearly none of these powers are translations, due to the fact that their linear part modifies at least one dimension.

Step 2. $s^{2}(z)=z+w$, since for $i=1, \ldots, 2 n, b_{i}-b_{2 n+1-i}=1 / 2$, and so $s$ does not have order 2 on $A^{\prime}$. Neverthless, since the linearity of $S$ and the fact that $S(w)=w$ imply that $s(z+w)=s(z)+w$, $s$ descends to an automorphism of $A$ of order exactly 2 .

Step 3. We have

$$
r s(z)=z M+b^{\prime}
$$

where

$$
M=\left[\begin{array}{ccccc}
1 & 0 & \ldots & 0 & 0 \\
\vdots & & I & & \vdots \\
0 & 0 & \ldots & 0 & -1
\end{array}\right], \quad I=\left[\begin{array}{cccc}
0 & \ldots & 0 & -1 \\
0 & & -1 & 0 \\
\vdots & -1 & & \vdots \\
-1 & \ldots & 0 & 0
\end{array}\right]
$$

and $b^{\prime}=\left(-b_{2 n}, b_{1}, \ldots, b_{2 n-1}, \frac{1}{4 n}\right)$. 
Hence, by simple computations, we have that

$$
\begin{aligned}
(r s)^{2}(z) & =z M^{2}+b^{\prime} M+b^{\prime} \\
& =z
\end{aligned}
$$

Also, because it was already shown for $r$ and $s$, it is also true that $r s(z+w)=r s(z)+w$, and so $r s$ descends to an automorphism of $A$ of order 2. Thus, we have an action of $D_{4 n}$ on $A$, since the orders of $r, s$ and $r s$ are precisely $4 n, 2$ and 2 , respectively.

Step 4. We claim that also the reflections in $D_{4 n}$ are not translations, noticing that, since dihedral groups representing even polygons have two conjugacy classes of reflections, those of $s$ and $r s$, it suffices to observe that these two transformations are not translations.

In the next step we show that they both act freely on $A$.

Step 5. It is rather immediate that $r s$ acts freely in $A$, since $r s(z)=z$ in $A$ is equivalent to the difference

$$
r s(z)-z=\left(-b_{2 n},-z_{2 n}-z_{2}+b_{1}, \ldots,-z_{2}-z_{2 n}+b_{2 n-1},-2 z_{2 n+1}+\frac{1}{4 n}\right)
$$

being a multiple of $w$ in $A^{\prime}$, but this is absurd since the only multiples of $w$ are zero and $w$ itself, while $-b_{2 n}=\tau / 2 \neq 0,1 / 2$.

On the other hand, $s$ acts freely on $A$ because $s(z)=z$ in $A$ is equivalent to the difference

$$
s(z)-z=\left(-z_{2 n}-z_{1}+b_{1},-z_{2 n-1}-z_{2}+b_{2}, \ldots,-z_{1}-z_{2 n}+b_{2 n},-2 z_{2 n+1}\right)
$$

being a multiple of $w$ in $A^{\prime}$, but the first and $2 n$-th coordinate of multiples of $w$ are equal, while here the difference between them is $b_{1}-b_{2 n}=1 / 2 \neq 0$.

\section{$3 \quad$ Using any dihedral group}

Notice that, although the previous construction is somewhat restrictive because it works with very specific dihedral groups, since it is true that $D_{n} \subseteq D_{k n}$ for all $n, k \in \mathbb{N}$, we can always make a bigger dihedral group act on some variety using the method above, and then restrict ourselves to a smaller one. So we have the following corollary:

Corollary 3.1. For all $n \in \mathbb{N}$, there exists a free action of the dihedral group $D_{n}$ of order $2 n$ on some Abelian variety of dimension $\frac{\operatorname{lcm}(4, n)}{2}+1$ that contains no translations.

It is interesting to observe that, although the relation is far away from being one-to-one, we have shown that for every odd dimension there is an Abelian variety and a dihedral group acting on it, 
and for every dihedral group there is an Abelian variety of odd dimension on which it acts, in a way that the quotient forms a Generalized Hyperelliptic Variety.

\section{Acknowledgement}

I would like to thank professors Robert Auffarth and Giancarlo Lucchini Arteche for introducing me to this topic. 


\section{References}

[1] R. Auffarth and G. Lucchini Arteche, "Smooth quotients of complex tori by finite groups", Preprint (2021). arXiv:1912.05327.

[2] F. Catanese and P. Corvaja, "Teichmüller spaces of generalized hyperelliptic manifolds", Complex and symplectic geometry, Springer INdAM Ser. 21, Springer, Cham, 2017, pp. 39-49.

[3] F. Catanese and A. Demleitner, "The classification of hyperelliptic threefolds", Groups Geom. Dyn, vol. 14, no. 4, pp. 1447-1454, 2020.

[4] F. Catanese and A. Demleitner, "Hyperelliptic threefolds with group $D_{4}$, the dihedral group of order 8", Preprint (2018), arXiv:1805.01835.

[5] E. C. Mistretta, "Holomorphic symmetric differentials and parallelizable compact complex manifolds", Riv. Math. Univ. Parma (N.S.), vol. 10, no. 1, pp. 187-197, 2019.

[6] K. Uchida and H. Yoshihara, "Discontinuous groups of affine transformations of $C^{3}$ ". Tohoku Math. J. (2), vol. 28, no. 1, pp. 89-94, 1976. 